\title{
Real-time follow-up of multi-messenger alerts with the ANTARES neutrino telescope
}

\author{
The ANTARES Collaboration ${ }^{* *}$ \\ \$ http://antares.in2p3.fr/Collaboration/index2.html \\ E-mail: dornic@cppm.in2p3.fr
}

\begin{abstract}
By constantly monitoring at least one complete hemisphere of the sky, neutrino telescopes are well designed to detect neutrinos emitted by transient astrophysical events. Real-time searches for neutrino candidates coincident with Swift and Fermi gamma-ray bursts, IceCube high energy neutrino events and gravitational wave (GW) candidates observed by LIGO/Virgo are performed with the ANTARES telescope. By requiring temporal coincidence, this approach increases the sensitivity and the significance of a potential discovery. The latest results of these analyses will be presented, with emphasis on the results of the neutrino follow-up of IceCube very high-energy neutrino events and LIGO/Virgo GW public events during the run O3. In case of a coincident detection, the position of the GW source on the sky would be largely constrained thanks to the better angular accuracy of ANTARES compared to the GW detectors, bringing valuable information for subsequent electromagnetic follow-ups. In case of no coincidence, an upper limit on the neutrino fluence and the total energy emitted in neutrinos will be provided for each studied alert.
\end{abstract}

Corresponding authors: D. Dornic ${ }^{\dagger 1}$, A. Coleiro ${ }^{2}$, M. Colomer Molla ${ }^{2,3}$, A. Kouchner ${ }^{2}$, T. Pradier $^{4}$

${ }^{1}$ Aix Marseille Univ, CNRS/IN2P3, CPPM, Marseille, France.

2 APC, Univ Paris Diderot, CNRS/IN2P3, CEA/Irfu, Obs de Paris, Sorbonne Paris Cité, France.

${ }^{3}$ IFIC, Instituto de Física Corpuscular (CSIC - Universitat de València) c/Catedrático José Beltrán, 2 E-46980 Paterna, Valencia, Spain.

${ }^{4}$ Université de Strasbourg, CNRS, IPHC UMR 7178, F-67000 Strasbourg, France.

36th International Cosmic Ray Conference -ICRC2019-

July 24th - August 1st, 2019

Madison, WI, U.S.A.

*for collaboration list see PoS(ICRC2019)1177

†speaker. 


\section{Introduction}

Time-domain astronomy has received a considerable boost in recent years with the development of wide-field-of-view instruments, optimized communication strategies and low latency alerts. This domain offers unique ability to study extreme physics, to track cataclysmic phenomena like the birth of stellar mass black holes or the mergers of neutron stars, to probe distant regions of the Universe, and to identify candidate sources for multi-messenger astrophysics. These explosive events can release enormous amounts of energy both in electromagnetic radiation and in non-electromagnetic forms such as neutrinos and gravitational waves. Multi-messenger astronomy has emerged as a major new field in astronomy during the last years. Major breakthroughs were the first observed coincidence of gravitational waves and electromagnetic signals over a huge wavelength regime from a merger of two neutron stars [1] and the detection of gamma rays and neutrinos from a flaring blazar [2]. In the LIGO/VIRGO run $\mathrm{O} 2$ and the ongoing run $\mathrm{O} 3$, few tens of gravitational waves have been detected so far.

In particular, neutrinos represent unique probes to study high-energy cosmic sources. They are neutral, stable and weakly interacting. Contrary to cosmic rays (CRs), they are not deflected by the magnetic fields and unlike high-energy photons, they are not absorbed by pair productions with cosmic microwave and infrared backgrounds. A HEN diffuse flux of cosmic origin has been identified by the IceCube telescope in 2012 [3] and confirmed later with all neutrino flavours [4]. The major result is undoubtedly the detection of a high-energy neutrino detected by IceCube on September 22, 2017, likely associated with a flare of the blazar TXS 0506+056 seen by gammaray, X-ray, optical, infrared, and radio telescopes [2]. If confirmed in the future, this association may mark the real birth of the high-energy neutrino astronomy. ANTARES is also detecting a mild excess of neutrino events in both track and cascade channels at very high energy [5].

In this context, multi-messenger approaches consisting in simultaneously looking for the same sources with both neutrino telescopes, gravitational wave interferometers and/or multi-wavelength facilities constitute a privileged way of locating HEN/CR sources and thus further understanding the acceleration mechanisms at play in these sources. Search for transient sources of HEN is promising since the short timescale of emission drastically reduces the background level, mainly composed of atmospheric muons and neutrinos and consequently increases the sensitivity and discovery potential of neutrino telescopes.

In particular, dedicated real-time analyses have been developed in the ANTARES Collaboration to look for neutrino events in both time and space coincidence with transient events announced by public alerts distributed through the Gamma-ray Coordinated Network (GCN) or by private alerts transmitted via special channels. Then, more optimized offline analyses are performed to search for neutrino counterparts to catalogued transients.

After showing the main characteristics of the ANTARES on-line acquisition and analysis frameworks in Section 2, the real-time follow-up analyses performed with ANTARES after the detection of transient events of gravitational wave events by LIGO/Virgo, high-energy neutrino candidates by IceCube and gamma-ray bursts by Swift, Fermi and INTEGRAL are described in Section 3, Section 4 and Section 5, respectively. 


\section{Real-time acquisition and analysis framework of ANTARES}

The data acquisition system of the ANTARES detector is based on the "all-data-to shore" concept [6]. The time and charge amplitude of all the photomultiplier signals above a threshold of 0.3 photoelectrons are sent to a computing farm onshore for processing $(\approx 1.5 \mathrm{~s})$. Filters based on combination of local clusters of hits are applied on shore to select the physics events among the raw dataset dominated by hits due to the optical background produced by bioluminescence and radioactive decay of ${ }^{40} \mathrm{~K}$. The selected events are finally kept for online and offline reconstruction as described in [7]. In particular, a fast and robust online algorithm reconstructs tracks in nearly real time [8]. The remaining sample of events is then sent to a more accurate reconstruction algorithm [9] which improves the angular resolution of each event in less than $5 \mathrm{~s}$. Both algorithms use an idealised geometry of the detector that does not take into account the dynamical positioning of the optical modules due to sea current variations. These online data are routinely used to look for neutrino counterparts to various astrophysical transient events whose detection is notified directly to ANTARES through both VOEvents ${ }^{1}$ and/or GCN socket and also to send alerts after the detection of interesting neutrino events (as presented in another proceeding [10]).

Figure 1 (left) shows the data-taking efficiency of the ANTARES detector since 2008. One of the main characteristics of the neutrino telescopes is the very high duty cycle, for ANTARES it is higher than $95 \%$. Figure 1 (right) illustrates the typical delay of about $5 \mathrm{~s}$ for the reconstruction of the track events. This enables to trigger very low latency neutrino alerts and real-time analysis after external astrophysical alerts. The latest results of these studies are presented hereafter.
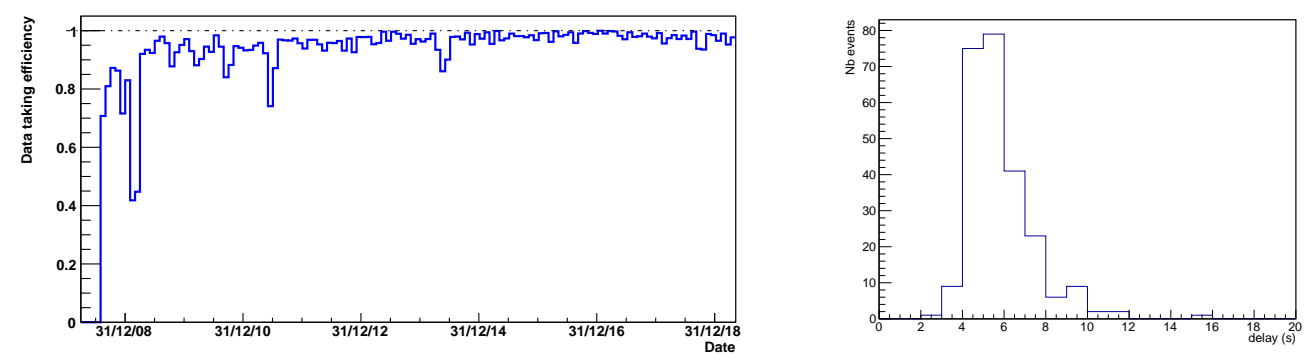

Figure 1: (Left) Average data-taking efficiency of the ANTARES detector. (Right) Typical delay between the time of the neutrino and the end of the two track reconstructions.

\section{Follow-up of gravitational wave events}

During the first observing run $\mathrm{O} 1$ in 2015, three gravitational waves (GW) coming from binary black hole (BBH) mergers have been detected by LIGO for the first time [1]. As the online analysis was not ready for these events, only offline analyses have been performed by the ANTARES Collaboration [11]. About one year later, during the second observing run O2 (November 30th, 2016 to August 25th, 2017), the upgraded LIGO and VIRGO detectors have detected GW from a total of seven binary black hole mergers (plus 3 additional sources found in the offline analyses) and

\footnotetext{
${ }^{1}$ standardized language adopted by the International Virtual Observatory Alliance.
} 
a binary neutron star (BNS) inspiral GW170817. Only for this last event, electromagnetic counterparts have been identified as a short gamma-ray burst followed by a kilonova [12]. During the run $\mathrm{O} 2$, the LIGO/VIRGO Collaboration has triggered 15 alerts identified by online analysis using a loose false-alarm-rate threshold of one per month. These triggers were shared with partner collaborations who have signed memoranda of understanding with LIGO/Virgo for electromagnetic and neutrino follow-up. Each of these alerts were followed by the ANTARES neutrino telescope by searching for a potential neutrino counterpart. The online analysis consists in looking for $(i)$ temporal coincidences within a $\pm 500 \mathrm{~s}$ and a \pm 1 hour time windows around the GW alert [13] and (ii) spatial overlap between the $90 \%$ probability contour and the ANTARES visibility region at the time of the GW event. No up-going neutrino candidates temporally coincident with all the GW candidates were found with ANTARES. The results of the nearly real-time analyses have been transmitted to LIGO/Virgo follow-up community via the GCN [14]. The first goal of the neutrino follow-up is that the angular resolution of ANTARES $\left(\sim 0.4^{\circ}\right.$ at $\left.\sim 10 \mathrm{TeV}\right)$ compared to the size of the gravitational wave error box (a few hundreds of square degrees on the sky) offers the possibility to drastically reduce the size of the region of interest in case of a coincident neutrino detection. In general, the online analyses performed on each GW candidates are followed by a more optimised all-sky analyses [15]. The most recent results will be presented to this conference [16].

The third observing run O3 has started beginning of April 2019 with even more upgraded interferometers. Up to end of June 2019, 17 alerts have been distributed publicly, 14 confirmed astrophysical events and 3 retracted by LIGO/VIRGO after further investigations. Among the 14 events, 11 are BBHs, 1 NSBH and 1 BNS. In the recent analysis, S190510g, the 2nd BNS seems to have a terrestrial origin. The ANTARES Collaboration has performed real-time analysis for $12 \mathrm{GW}$ triggers. One trigger has not been analysed since the GW error box was too North for ANTARES while for the other event, ANTARES was in maintenance during the event. The main characteristics of the $17 \mathrm{GW}$ candidates and the results of the neutrino search are summarised in Table 1. For these new events, we have performed similar analyses as done for the run $\mathrm{O} 2$ events. Up to now, no up-going muon track neutrino has been found in time and space coincidence with each alert of run O3 [17]. Figure 2 illustrates the errors contours of of GW event S190602aq together with the ANTARES visibility at the time of the event.

\section{Follow-up of IceCube high-energy neutrinos}

IceCube is currently the largest neutrino telescope. Located at the geographic South Pole, it is composed of 86 detection lines distributed over a cubic-kilometer of ice. Since 2016, high-energy starting events (HESE, see e.g. [4]) and extremely high-energy track candidates (EHE) are received by the Astrophysical Multi-messenger Observatory Network (AMON, [18]) and distributed to the community via an alert of the $\mathrm{GCN}^{2}$. In June 2019, IceCube is providing in replacement two new very high energy track event samples: gold (with a probability to be astrophysical $p>50 \%$ ) and bronze ( $\mathrm{p}>30 \%)$ samples.

A coincident detection by both IceCube and ANTARES would be a significant proof of the astrophysical origin of these neutrino candidates and would point directly to the position of the

\footnotetext{
${ }^{2}$ https://gcn.gsfc.nasa.gov/amon.html
} 


\begin{tabular}{|c|c|c|c|c|c|c|}
\hline GW name & Type & $\begin{array}{c}\text { Latency } \\
(\mathrm{min})\end{array}$ & $\begin{array}{c}\text { Error box } \\
\left(\mathrm{deg}^{2}\right)\end{array}$ & $\begin{array}{c}\text { FAR } \\
\left(\mathrm{yr}^{-1}\right)\end{array}$ & $\begin{array}{c}\text { Coverage } \\
(\%)\end{array}$ & $\begin{array}{c}\text { GCN } \\
\text { number }\end{array}$ \\
\hline S190405ar & Retracted & & & & & \\
S190408an & BBH & 34.5 & 381 & $1 / 1.12 \mathrm{e}-10$ & $/$ & no analysis \\
S190412m & BBH & 60.9 & 156 & $1 / 1.89 \mathrm{e} 19$ & 9 & 24105 \\
S190421ar & BBH & 1247.5 & 1444 & $1 / 2.13$ & 52 & 24156 \\
S190425z & BNS & 42.8 & 7461 & $1 / 69384$ & $/$ & no analysis \\
S190426c & NSBH & 25.3 & 1131 & $1 / 1.63$ & 45 & 24271 \\
S190503bf & BBH & 36.2 & 448 & $1 / 19.37$ & 98 & 24387 \\
S190510g & Terrestrial & 82.3 & 1166 & $1 / 3.59$ & 55 & 24446 \\
S190512at & BBH & 51.8 & 252 & $1 / 16.67$ & 83 & 24516 \\
S190513bm & BBH & 27.4 & 691 & $1 / 84864$ & 55 & 24539 \\
S190517h & BBH & 35.8 & 939 & $1 / 13.35$ & 83 & 24581 \\
S190518bb & Retracted & 6.5 & & & & \\
S190519bj & BBH & 85.9 & 967 & $1 / 5.56$ & 34 & 24602 \\
S190521g & BBH & 6.3 & 765 & $1 / 8.34$ & 56 & 24628 \\
S190521r & BBH & 6.5 & 488 & $1 / 100.04$ & 30 & 24634 \\
S190524q & Retracted & 6.5 & & & & \\
S190602aq & BBH & 6.6 & 1172 & $1 / 16.67$ & 84 & 24719 \\
\hline
\end{tabular}

Table 1: Characteristics of the GW candidates distributed by LIGO/VIRGO during run O3. The coverage indicates the fraction of the $90 \% \mathrm{GW}$ error box falling in the visibility of the ANTARES neutrino telescope at the time of the event.

source in the sky. In this context, the ANTARES collaboration is performing a follow-up analysis of each IceCube event whose position is below the horizon of ANTARES (which could consequently yield to an up-going event at the time of the alert). Up to now, ANTARES has received 27 neutrino triggers from IceCube and has followed 11 alerts (7 HESE, 3 EHE and 1 gold). The rest of triggers was either retracted by the IceCube Collaboration or in the wrong hemisphere. No neutrino candidates were found compatible with one of the alerts within a cone of 3 degrees centered on the IceCube event coordinates and a time window of \pm 1 hour and an extended one of \pm 1 day. These non-detections have been used to derive preliminary $90 \%$ confidence level upper limits on the radiant neutrino fluence of these events of the order of $\sim 15 \mathrm{GeV} \mathrm{cm}^{-2}$ and $\sim 30 \mathrm{GeV} \mathrm{cm}^{-2}$ for the $\mathrm{E}^{-2}$ and the $\mathrm{E}^{-2.5}$ spectral models respectively (see Table 2). These results have been published as GCN circulars within some hours after the alerts [19].

\section{Follow-up of GRBs}

Transient astrophysical events are observed all over the electromagnetic spectrum. Gammaray bursts (GRB) are mainly detected by X-ray and gamma-ray satellites such as Swift, FermiGBM. Once a GRB is detected, an alert message is sent publicly via the GCN within few tens of seconds. ANTARES is able to react in real-time to this type of alerts. Only the bursts with their direction visible in the ANTARES horizon are analysed. A dedicated search for muon-track 


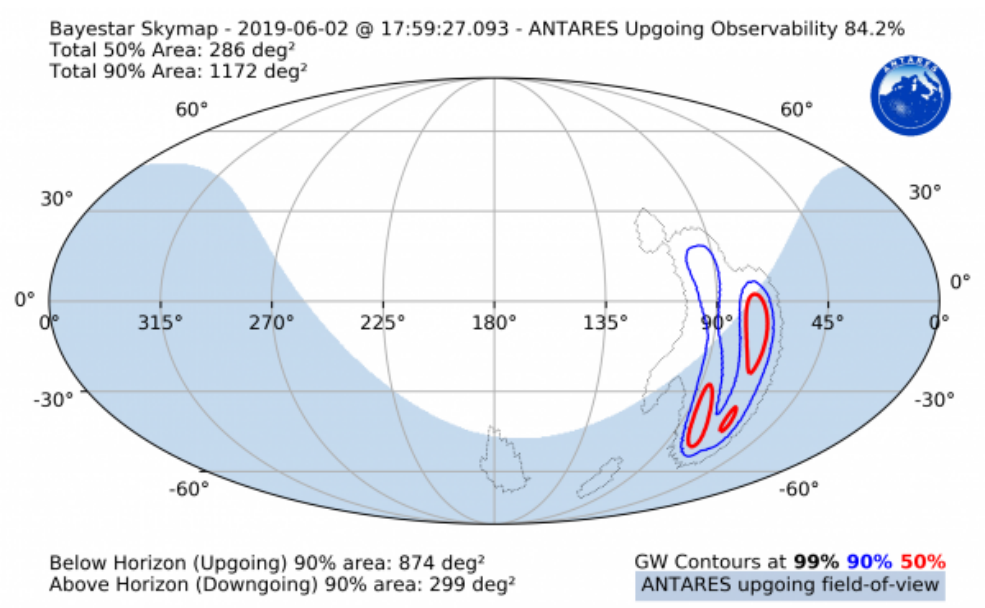

Figure 2: Skymap in equatorial coordinates showing the $99 \%$ (black), $90 \%$ (blue) and $50 \%$ (red) probability contours of S190602aq together with the ANTARES field-of-view at the event time (blue part of the map).

\begin{tabular}{|c|c|c|c|}
\hline IceCube event & Elevation (deg) & \multicolumn{2}{|c|}{ Fluence U.L. $\left(\mathrm{GeV} \mathrm{cm}^{-2}\right)$} \\
& & $d N / d E \propto E^{-2}$ & $d N / d E \propto E^{-2.5}$ \\
\hline IC160731A (EHE/HESE) & $-28^{\circ}$ & $14(2.8 \mathrm{TeV}-3.1 \mathrm{PeV})$ & $27(0.4-280 \mathrm{TeV})$ \\
IC160814A (HESE) & $-26^{\circ}$ & $16(2.9 \mathrm{TeV}-3.3 \mathrm{PeV})$ & $43(0.5-250 \mathrm{TeV})$ \\
IC161103A (HESE) & $-26^{\circ}$ & $13(3.8 \mathrm{TeV}-3.8 \mathrm{PeV})$ & $22(0.7-370 \mathrm{TeV})$ \\
IC170321A (EHE) & $-57^{\circ}$ & $16(2.5 \mathrm{TeV}-2.5 \mathrm{PeV})$ & $26(0.5-220 \mathrm{TeV})$ \\
IC170922A (EHE) & $-14^{\circ}$ & $15(3.3 \mathrm{TeV}-3.4 \mathrm{PeV})$ & $34(0.5-280 \mathrm{TeV})$ \\
IC171015A (HESE) & $-45^{\circ}$ & $14(2.7 \mathrm{TeV}-2.9 \mathrm{PeV})$ & $27(0.4-240 \mathrm{TeV})$ \\
IC180908A (EHE) & $-41^{\circ}$ & $18(2.4 \mathrm{TeV}-2.6 \mathrm{PeV})$ & $36(0.4-250 \mathrm{TeV})$ \\
IC190104A (HESE) & $-39^{\circ}$ & $16(3.2 \mathrm{TeV}-3.5 \mathrm{PeV})$ & $30(0.6-315 \mathrm{TeV})$ \\
IC190124A (HESE) & $-44^{\circ}$ & $15(3.1 \mathrm{TeV}-3.6 \mathrm{PeV})$ & $25(0.6-316 \mathrm{TeV})$ \\
IC190504A (HESE) & $-18^{\circ}$ & $16(3.1 \mathrm{TeV}-3.5 \mathrm{PeV})$ & $32(0.6-316 \mathrm{TeV})$ \\
IC190619A (gold) & $-19^{\circ}$ & $13(3.9 \mathrm{TeV}-3.9 \mathrm{PeV})$ & $33(0.7-320 \mathrm{TeV})$ \\
\hline
\end{tabular}

Table 2: Preliminary ANTARES fluence upper limits at 90\% C.L. for each IceCube neutrino candidate. For each fluence, the 5-95\% energy range is given.

neutrino counterparts in the optimized online dataset is performed in real-time within [-250 s; $+750 \mathrm{~s}]$ time window around the detection time and in a cone of max $\left[2^{\circ}\right.$,error box Fermi] radius centered on the GRB position (Figure 3). To ensure the quality of the data at the alert time, the detector stability is checked over several hours before the alert. This analysis is operational since beginning of 2014 and up to now $\approx 98 \%$ of the alerts have been processed. During this about 5 year of operation, there were 226 Swift and 536 Fermi-GBM bursts.

In case of a coincident neutrino detection, a dedicated offline analysis is run to confirm the result and compute its significance (expected to be higher than $3 \sigma$ in most of the cases). For example, dedicated offline analyses have been performed by the ANTARES Collaboration [20, 21, 

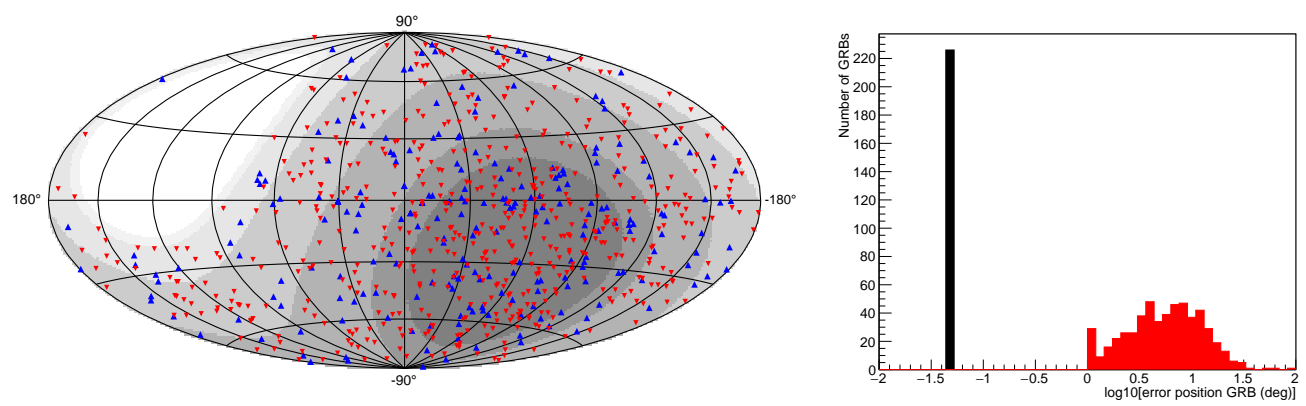

Figure 3: (Left) Skymap on galactic coordinates with the positions of the Fermi (red triangles) and Swift (black triangles) GRBs followed by ANTARES. The shade of grey indicates the ANTARES visibility. (Right) Error on the position of the GRBs detected by Swift (black) and Fermi-GBM (red).

22].

\section{Summary}

Multi-messenger astrophysics and in particular rapid follow-up of transient events have become one of the main domains in observational astrophysics and perhaps the most active. It has shown its power with the follow-up campaign of the binary neutron merger (GW170817 / GRB170817). The detection of a coincident neutrino with a typical localisation accuracy of a fraction of a degree to compare with the few to tens of degrees for gravitational waves, would allow us to study such phenomena some seconds or minutes after the trigger, uncovering so far nonobservable physical processes besides probing the long-sought role and importance of hadrons in that transient violent astrophysical events.

By simultaneously monitoring at least half of the sky and by its ability to reconstruct events in real-time, ANTARES is very well-suited to detect transient sources. The ANTARES Collaboration has developped a very rich real-time multi-messenger program that includes the follow-up in few tens of seconds of external electromagnetic and multi-messenger triggers (gamma-ray burst, fast radio burst, IceCube high-energy events, gravitational waves, etc) and to trigger electromagnetic follow-up of interesting neutrino candidates [10,23]. Only in 2019, this represents more than 100 alerts processed by the Collaboration.

\section{References}

[1] Abbott, B., Abbott, R., Abbott, T. D., et al. 2016a, PhRvX, 6, 041015; Abbott, B., Abbott, R., Abbott, T. D., et al. 2017d, PhRvL, 119, 161101.

[2] Aartsen, M., Ackermann, M., Adams, J., et al. 2018, Sci, 361, 147.

[3] Aartsen, M., Abbasi, R., Abdou, Y., et al. 2013a, PhRvL, 111, 021103; Aartsen, M., Abbasi, R., Abdou, Y., et al. 2013b, Sci, 342, 1242856.

[4] Aartsen M. G. et al. (IceCube), ApJ, 809(1), 98 (2015).

[5] Albert A. et al. 2018a, ApJL, 853, L7; Fusco L. et al. Pos(ICRC2019)891. 
[6] Aguilar J et al., (ANTARES Collaboration), Nucl. Instrum. Meth., A570 107 (2007).

[7] Ageron M et al., (ANTARES Collaboration), Astropart. Phys., 35:530-536 (2012).

[8] Aguilar J A et al., (ANTARES Collaboration), Astropart. Phys., 342011 652-662 (2011).

[9] Adrían-Martinez S et al., (ANTARES Collaboration), ApJ, 760:53 (2012).

[10] Dornic D et al. (ANTARES Collaboration), Pos(ICRC2019)871.

[11] Albert A. et al, (ANTARES Collaboration), Phys. Rev. D 96 (2017) 022005; Adrían-Martinez S et al., (ANTARES Collaboration), Phys. Rev. D 93 (2016) 122010.

[12] Abbott, B. et al, ApJL, 848 L12 (2017).

[13] Baret B et al., Astropart. Phys., 35 1:7 (2011).

[14] Ageron M., Baret B., Coleiro, A. Dornic D, Kouchner A. \& Pradier T., (ANTARES Collaboration), GCN\#20370, 20487, 20517, 20621, 20704, 20751, 20765, 20866, 21066, 21223, 21433, 21479, 21522, 21601, 21631, 21659, 21696, 21769.

[15] Albert A. et al, (ANTARES Collaboration), Eur. Phys. J. C 77, 911 (2017);Albert A. et al, (ANTARES Collaboration), ApJL 850 L35 (2017)

[16] Colomer Molla M. et al.,(ANTARES Collaboration), Pos(ICRC2019)856.

[17] Ageron M., Baret B., Coleiro, M. colomer-Molla, A. Dornic D, Kouchner A. \& Pradier T., (ANTARES Collaboration), GCN\#23793, 24105, 24156, 24271, 24387, 24446, 24516, 24539, 24581 , 24602, 24628, 24634, 24719.

[18] Smith M W E et al., Astropart. Phys., 45:56-70 (2013).

[19] Dornic D \& Coleiro A, (ANTARES Collaboration), ATeL\#9324, 9440, 9715, 10189 , 10773, 10854, $12024,12359,12423,12731,12878$.

[20] Albert A et al., (ANTARES Collaboration), Mon. Not. Roy. Astron. Soc., 469906 (2017).

[21] Adrían-Martinez S et al., (ANTARES Collaboration), Astron. Astrophys., 559 (2013).

[22] Adrían-Martinez S et al., (ANTARES Collaboration), Eur. Phys. J. C, 77:20 (2017).

[23] Adrían-Martínez S et al., (ANTARES Collaboration), JCAP, 02:62 (2016). 